\title{
Combined scatter diagrams of sheath thickness and fibre calibre in human sural nerves: changes with age and neuropathy
}

\author{
RL FRIEDE, W BEUCHE \\ From the Department of Neuropathology, University of Göttingen, Federal Republic of Germany
}

SUMMARY A computer-assisted method permits collection of large numbers of measurements of fibre profiles in electron micrographs of human sural nerve biopsies. The method is based on simultaneous demonstration of fibre calibre and of sheath thickness in terms of the g-ratio (quotient axon diameter/fibre diameter), and on the recalculation of all parameters for circular fibre profiles. The fibres of small and large diameters of human sural nerves were found to form separate populations with distinctly different trends for sheath thickness and also different patterns of maturation. Preliminary data in neuropathy show that this method gives a better distinction of the extent of damage to either of the two populations; it also permits assessment of the number of regenerating or remyelinating fibres.

Evaluation of sural nerve biopsies customarily involves an assessment of the frequencies of myelinated fibres of different diameter ranges; such observations may be verified by computer-assisted calibre histograms.' Assessment of sheath thickness usually remains subjective, restricting observations to fibres with gross disproportions. In the present paper we report scatter diagrams of sheath thickness and fibre calibre in human sural nerve biopsies obtained from electron micrographs using a new computer-assisted method. Such scattergrams of the g-ratio (quotient axon diameter/fibre diameter) permit a simultaneous assessment of a fibre's calibre and of the thickness of its sheath.

The histometric curves reported here add detail to the existing knowledge on sural nerve fibre populations. They show, in particular, that the nerve is composed of two separate fibre populations which differ not only in calibre but also in sheath proportions. These differences are concealed in histograms showing only calibres. The main thrust of this paper is the documentation of the changes in these populations with age. Some data on neuropathy were

Address for reprint requests: Reinhard L Friede, M.D., Department of Neuropathology, University of Göttingen, Robert-KochStr. 40, D-3400 Göttingen, FRG.

Received 12 June 1984 and in final revised form 5 December 1984. Accepted 15 December 1984 added to obtain a preliminary view of the method's potential for assessing pathological changes in biopsy specimens.

\section{Materials and methods}

Sural nerve biopsy specimens were selected from the department's file of Araldite-embedded nerves covering a period of 10 years. A detailed description of the method is given in a separate publication. ${ }^{2}$ Briefly, the entire profile of the nerve was mapped with micrographs taken of every grid opening with a Zeiss EM10 electron microscope at a final magnification of $3400 \times$. This provided randomised sampling of the entire nerve. The sampling method was slightly biased toward small calibre fibres, as fibres of small calibre are less likely to be partially covered by grid bars than fibres of large calibre. That bias, however, was a constant factor for the entire material. The inner and outer surfaces of the sheaths were traced manually with a cursor and were measured using a Kontron videoplan. A new type of computer software developed for these measurements ${ }^{2}$ determined four primary parameters (circumference of axon, circumference of sheath, area of axon, and area of sheath) and calculated six secondary parameters, including the non-circularity of the axons (cross-sectional area of the axon measured/area of a full circle having the same circumference), the recalculation to circular profiles of the diameters, the sheath thickness, the various areas, and the g-ratio of the fibres. Circular diameters shown by this program are the diameters of circles of equivalent circumference. This is an important deviation from the usual Kontron videoplan program which gives diameters for equivalent areas. 


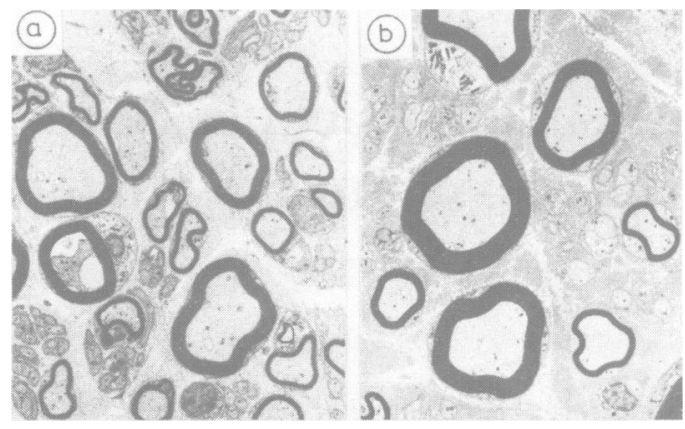

Fig 1 Representative fields of sural nerve of patients aged (A) 1 year and $(B) 27$ years. Abnormal fine structure is evident. $(\times 3400$. $)$

Routine evaluation of a nerve involved printing of the following scatter diagrams: g-ratio versus circular axon diameter; non-circularity factor versus circular axon diameter; sheath thickness versus circular axon diameter; the area of myelin versus circular axon area; and the area of myelin versus circular fibre diameter. The scattergrams of the g-ratio shown here were found to be the most informative sets of data, the rest being used mainly for comparative purposes. Referring sheath thickness to the diameter of the circular axon profile is merely a mathematical extension of measuring fibres in terms of axon circum-

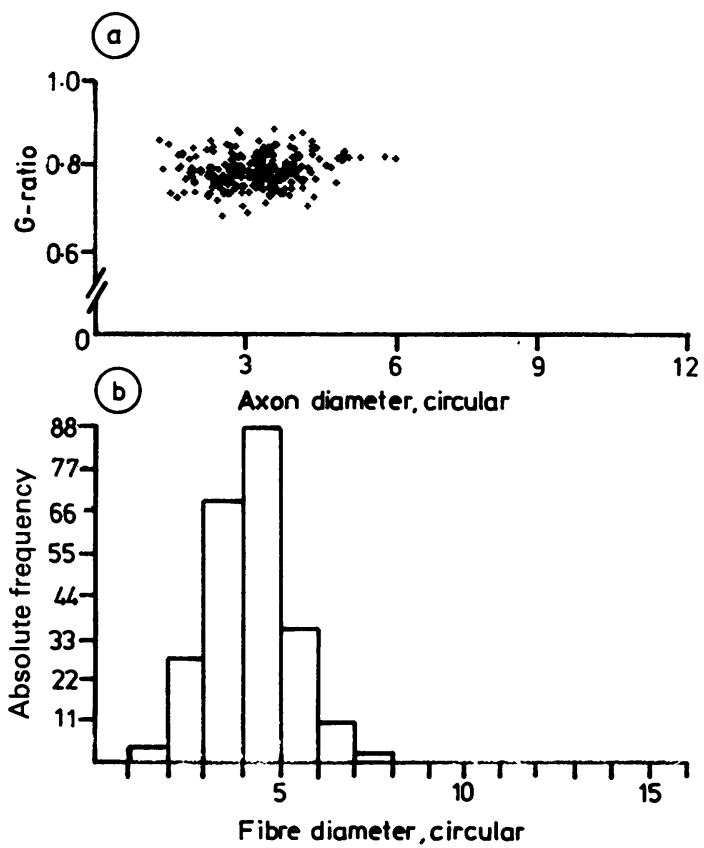

Fig 2 (a) Scatter diagram of sheath thickness (g-ratio: quotient axon diameter/fibre diameter) and (b) calibre histogram of a one-month-old child. ference ${ }^{3}$ which gives the most appropriate parameter of axon calibre. ${ }^{4}$ Each set of data on the g-ratio was accompanied by a parallel set of data on non-circularity to assess the degree of fibre deformation present in that given sample. One also has the option of printing the g-ratio versus axon area if one does not wish to correct for noncircularity. We found no advantage of using axon area in the present study as the only observable effect of not correcting for non-circularity was the introduction of random scatter, varying from one sample to the next and depending mainly on conditions of tissue preservation.

\section{Results}

Sural nerve biopsy specimens of strictly normal infants and children are not available for obvious reasons. To obtain such material we screened our files of biopsies for cases that had been diagnosed as being free of pathological changes when originally read. These were reviewed; only those showing excellent tissue preservation and no evidence whatsoever of pathologic changes were selected (fig 1). Collecting the prints for measurements involved a complete electron microscopic review of the nerve; no pathological changes of fine structure were seen. We felt justified in considering such nerves as being normal even though we had no material of teased fibres for additional verification. Our study benefited from a rather liberal clinical indication of nerve biopsy which had often been used as a last $\stackrel{\mathbb{Q}}{\square}$ resort even if there was no clinical evidence of neuropathy.

A method of trial and error was used to subdivide the material into age classes. This was done by superimposing the individual scattergrams in various combinations. Age groups were assembled on the basis of nearly identical scattergrams. Furthermore, if all the scattergrams for a given age group were identical, this was considered additional evidence of normality. These curves, moreover, were similar to those obtained in laboratory animals. ${ }^{2} \mathrm{~A}$ few cases were eliminated; these had been thought to be normal when the tissue was reviewed, but their scatter diagrams showed subtle variations suggesting pathological change. As the residue of this process of elimination, we had 16 normal nerve scattergrams.

Scattergrams of sheath thickness (g-ratio) may be read and understood on their own merits; to facilitate comparison, they are shown here along with the traditional type of calibre histograms. It will be noted that the calibre histograms are printed for diameters of circular fibres, while g-ratio scattergrams are printed for diameters of circular axons, accounting for differences in calibre ranges. When translating the data obtained from embedded material into in vivo dimensions, a shrinkage factor of 0.7 

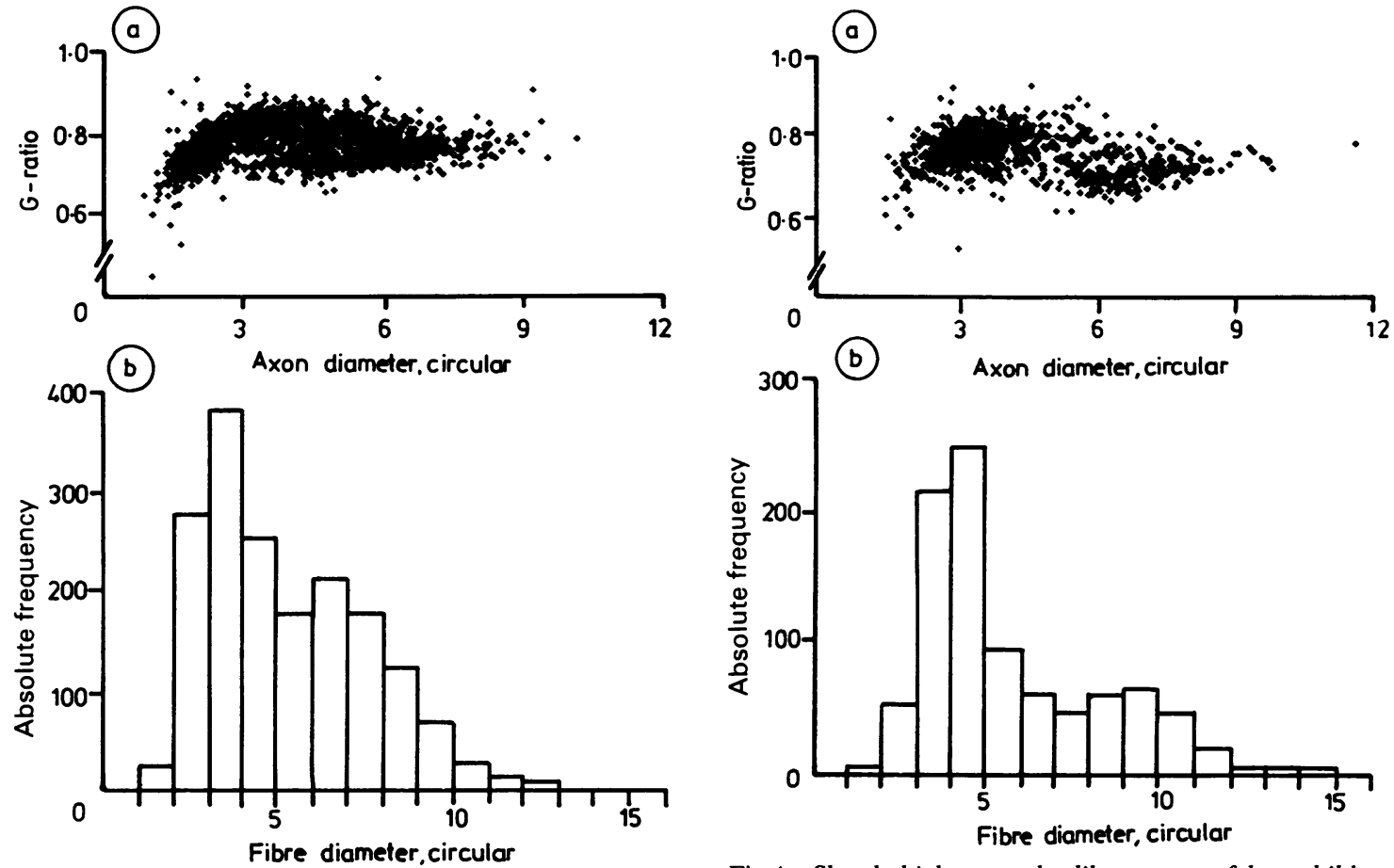

Fig 3 Sheath thickness and calibre ranges of three infants aged 6 to 12 months.

should be applied as determined in previous studies using similar material.

\section{Changes with age in the human sural nerve}

Although the changes during growth are continuous, one could divide our 16 cases into five age groups: below 6 months, 6 to 12 months, 2 to 4 years, 8 to 22 years, and adults.

Only one specimen was available for the below 6 months' age group: that of a one-month old infant. Axon calibres were small (mean $4 \cdot 18 \mu \mathrm{m}$ ), and the rather thin sheaths of these fibres (g-ratio $0 \cdot 80$ ) formed a homogenous cluster (fig 2). The calibre histograms, accordingly, showed a single peak. It was not possible to ascertain whether this cluster contained only one or both of the two populations found later on. One had the impression, however, that most of the fibres found at this age were destined to become fibres of large calibre, and that the population of small calibre fibres entered the diagrams between one and six months.

Infants between 6 and 12 months (three patients; 6, 12 and 12 months old) showed a different scattergram in which an early separation of calibre was apparent. A substantial part of the population of

Fig 4 Sheath thickness and calibre ranges of three children aged 2 to 4 years.

fibres of small calibre had even thinner sheaths (higher g-ratios) than the fibres found at one month, which supports the assumption that at least some of the small calibre fibres had myelinated between one and six months. The fibres of large calibre had grown substantially, from a maximum of $6 \mu \mathrm{m}$ at one month to $9 \mu \mathrm{m}$ at 6 to 12 months (fig 3). There was still so much overlap between the calibre ranges of the two populations that their existence was only hinted at in the calibre histograms.

In children aged 2 to 4 years (three patients; 2,2 and 4 years old), there was a clear-cut wide separation between the two fibre populations (fig 4). There had been relatively little change in the small calibre fibres, but the fibres of large calibre had grown both in calibre and in sheath thickness, thus moving their position in the scattergram to the right and down. The calibre histograms began to reflect this change without being able to show the different sheath proportions of the two populations.

Superimposition of the remaining two age groups (figs 5,6 ), the 8 to 22 -year olds (five patients; $8 \cdot 2$, $10,12,17$ and 22 years old), and the adults (four patients; 30, 40, 50 and 53 years old) showed very similar patterns. On close inspection, there was 

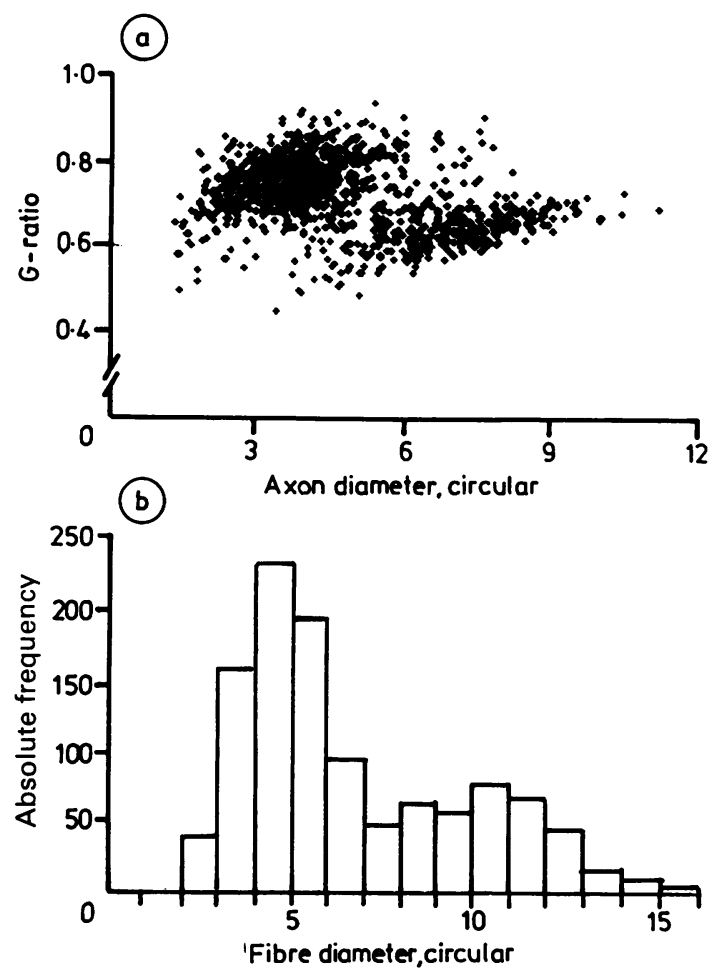

Fig 5 Sheath thickness and calibre ranges of five patients aged 8 to 22 years.

some change in the shape of the scattergrams of the thinly myelinated fibres of small calibre. Also, the regression for the large calibre fibres was slightly lower for adults, but the difference was minimal as shown in the table. We had kept these groups separate to test whether the wear and tear of advancing age or the summation effect of minor trauma, respectively, would be evident. There was no such difference for the small number of cases studied here. The structural organisation of the nerve evidently approaches a stable situation during the second decade of life.
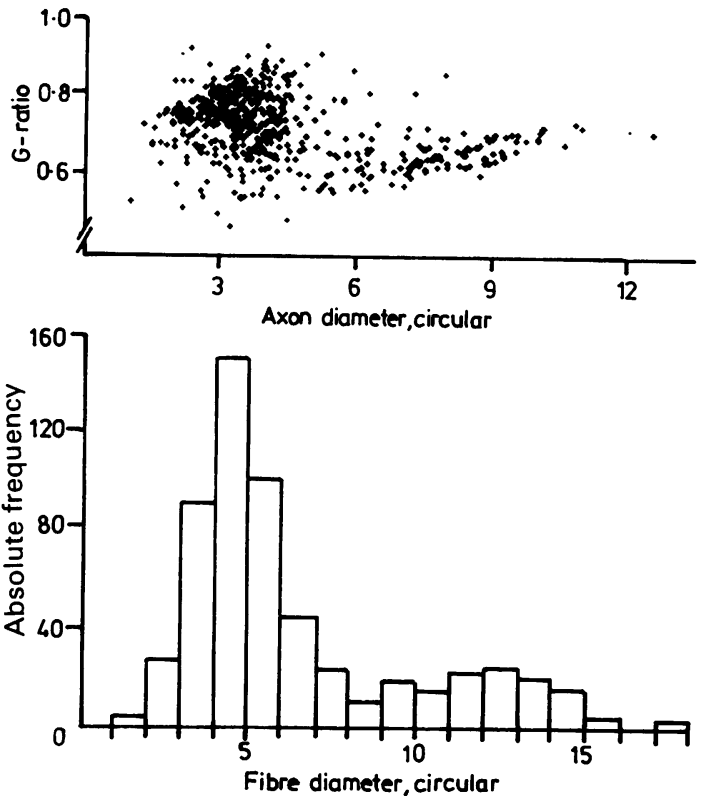

Fig 6 Sheath thickness and calibre ranges of four patients aged 30 to 53 years.

The shapes of scattergrams of the g-ratios are too irregular to justify calculation of an unqualified mean value for the thickness of sheaths at a givent age, and neither do they define a clear-cut general regression for sheath thickness with fibre calibre? The patterns found here were quite similar to theo ones obtained with this method in several animal. species as discussed later. The relative frequencies of the two populations may be estimated by counting the fibres on either side of a regression line fitted approximately at the zone of lowest fibre density between the two populations. The thinly myelinated fibre population of small calibre comprised, on average, $76 \%$ of the population. This value is only a rough approximation, as the regression line had to be fixed by hand, and also because of the sampling

Table 1 Mean sheath thickness ( $g$-ratio) at various ages for fibres of 3 to 4 um and 6 to 7 um axon diameter

\begin{tabular}{|c|c|c|c|c|}
\hline \multirow[t]{2}{*}{ Age group } & \multicolumn{2}{|c|}{ Axon diameter } & \multicolumn{2}{|c|}{ Axon diameter } \\
\hline & $3-4 \mu m$ & $n$ & 6-7 $\mu \mathrm{m}$ & $n$ \\
\hline $\begin{array}{l}\text { Group A below } 6 \text { months } \\
\text { Group B } 6 \text { to } 12 \text { months } \\
\text { Group C } 2 \text { to } 4 \text { years } \\
\text { Group D } 8 \text { to } 22 \text { years } \\
\text { Group E adults } \\
\text { Patient } 1 \\
\text { Patient } 2 \\
\text { Four alcoholics }\end{array}$ & $\begin{array}{l}0.80 \pm 0.04 \\
0.81 \pm 0.04 \\
0.79 \pm 0.04 \\
0.76 \pm 0.06 \\
0.75 \pm 0.08 \\
0.79 \pm 0.05 \\
0.81 \pm 0.07 \\
0.76 \pm 0.08\end{array}$ & $\begin{array}{l}(364) \\
(329) \\
(284) \\
(205)\end{array}$ & $\begin{array}{l}\text { (Mean diame } \\
0.77 \pm 0.03 \\
0.73 \pm 0.04 \\
0.66 \pm 0.09 \\
0.65 \pm 0.07 \\
0.72 \pm 0.06 \\
0.64 \pm 0.07 \\
0.65 \pm 0.09\end{array}$ & $\begin{array}{l}35 \mu \mathrm{m} \text { for all fibres) } \\
(162) \\
(80) \\
(107) \\
(22)\end{array}$ \\
\hline
\end{tabular}




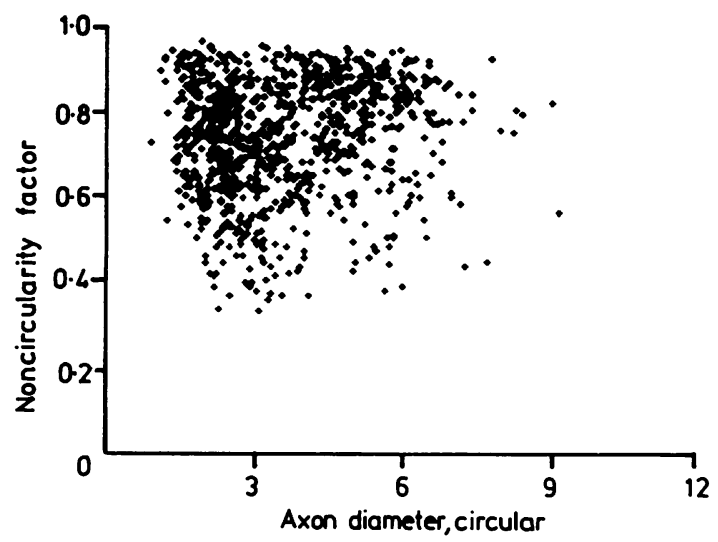

Fig 7 Scatter diagram of fibre shrinkage in the biopsy of a 20-year-old patient. The non-circulatory factor defines the quotient axon area measured/area of a circle having the axon's circumference.

bias described in Materials and methods. It seems safe, however, to assume that about two thirds of the human sural fibre population belong to the thinly myelinated fibres of small calibre. One may document the developmental changes for each of the two fibre populations by using a computer window for fibres 3 to $4 \mu \mathrm{m}$ and for fibres 6 to $7 \mu \mathrm{m}$. These calibre ranges are representative of two fibre classes throughout growth. This method (table) showed increase in sheath thickness for both fibre classes, but the increase was smaller for the thinly myelinated fibres of small calibre than for the others.

Each set of data came with a scattergram of fibre shrinkage (non-circularity factor). A representative sample is shown in fig 7. The scatter shown in this diagram is much greater than that found in nerves of experimental animals fixed under carefully controlled conditions. The latter usually show noncircularity factors in the range of $0 \cdot 8$, while biopsy material had non-circulatory factors near 0.5 or lower. The program provides the option to print data for area of axoplasm without correction for fibre shrinkage. This resulted in introducing large, irregular scatter, commensurate to that shown in fig 7. The most accurate standard of reference, therefore, was to print scattergrams corrected for circular profiles with additional data on the degree of circularity available for interpretation.

\section{Pathological scattergrams}

A preliminary sampling of abnormal scattergrams is shown here to indicate the type of deviation that one may expect to find in pathological specimens. The scattergram in fig 8 a was obtained from a 29 -year-

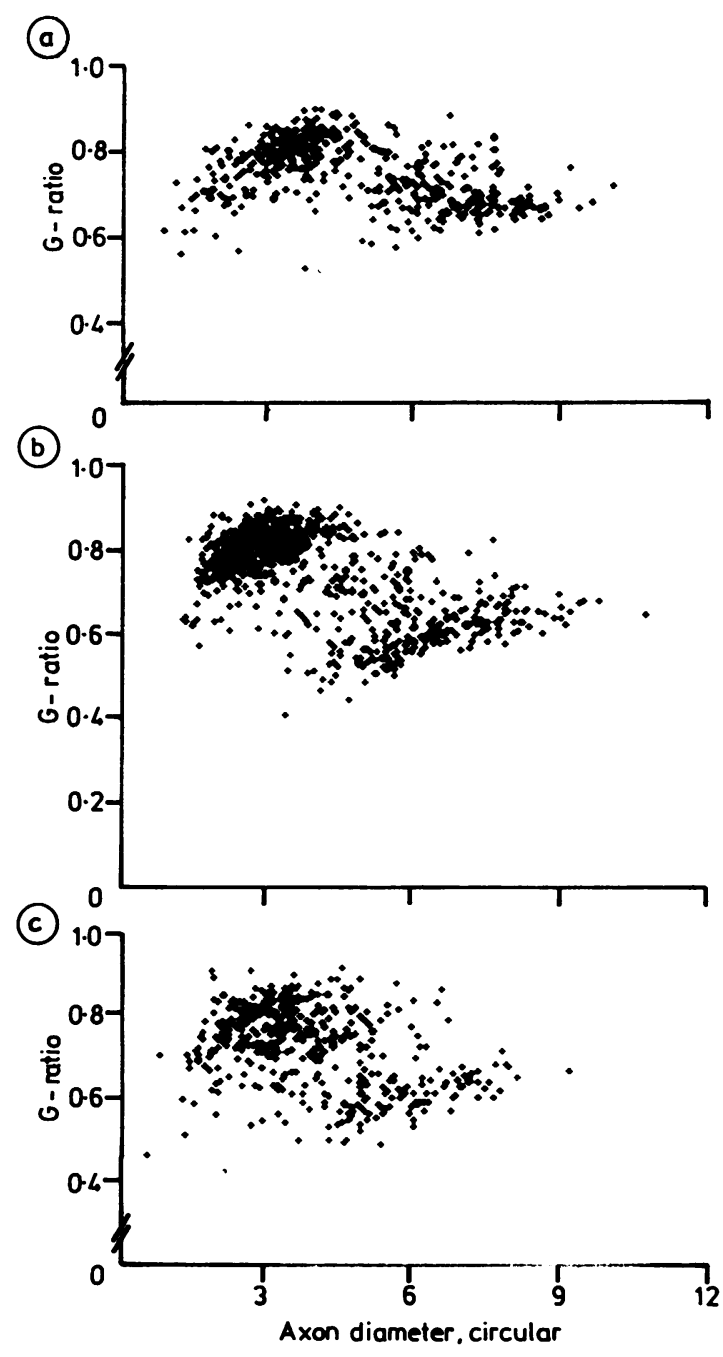

Fig 8 Abnormal scatter diagrams of sheath thickness $(a, b)$ in two patients with neuropathy and (c) in four patients with alcoholic neuropathy. Higher than normal g-ratios which mean thinner sheaths than normal $(a, b)$ may indicate regeneration.

old woman suffering from a painful, presumably para-infectious neuropathy from which the patient subsequently recovered. The nerve biopsy was read as being without pathological change. Compared with normal adults (fig 6), there are higher g-ratios. The thinly myelinated fibres of small calibre are shifted to the left upper quadrant and they are arranged in a shape similar to that seen in 2- to 4 -year-old infants (fig 3 ). The medium calibre fibres are reduced "from the bottom upwards" and there is 
an uncommon scattering of fibres between the two groups. The scattergram in fig $8 \mathrm{~b}$ was obtained from a 76-year-old woman whose biopsy was read as showing slight neuropathic changes. The clinical diagnosis presumed a nutritional disorder. This scattergram has the thinly myelinated fibres of small calibre still more displaced toward the left upper corner than in fig $8 \mathrm{a}$, but there is no concurrent change in the large calibre fibres so that the two groups lie much farther apart than normal.

Figure 8c, finally, shows the superimposed scattergrams of four patients with severe alcoholic polyneuropathy. In two patients the changes were so advanced that only a few fibres entered the compound scattergram, but these lay within the range of the rest. The "upward shift" seen in figs $8 \mathrm{a}$ and $8 \mathrm{~b}$ is not detectable. The largest fibres have disappeared but the proportion between the thinly myelinated fibres of small calibre and the fibres of large calibre was only slightly changed, being approximately $2: 1$.

\section{Discussion}

Scatter diagrams of the g-ratios of normal sural nerves of adolescents and adults showed a clear-cut separation of two populations of calibre ranges of myelinated fibres. The calibres of these two classes overlapped slightly, but the populations were set apart by different trends in the proportions between axon calibre and sheath thickness. The separation of the two populations was the more distinct the greater the number of measurements. If only small numbers of measurements are available, one may obtain the erroneous impression of a quasi linear increase of sheath thickness with axon calibre having a considerable degree of scatter. ${ }^{5}$ If that were true, there should be a constant g-ratio for all fibre calibres.

The sharp separation of the two fibre classes was at variance with much of the previous literature. Sheath thickness was generally thought to increase continuously with fibre calibre. Controversy pertained only to the question whether a curve defining that relationship was a straight line,$^{36}$ or a parabola, ${ }^{7}$ or whether the largest fibres would behave differently from the rest. ${ }^{8}$ The present data on sural nerves agree with the data of Boyd and Kaluy and with the results obtained with our method in the sciatic nerves of frogs, mice, rats and cats, and rabbits. ${ }^{2}$ All of these species have populations of thinly myelinated fibres of small calibre forming a cluster in the left upper quadrant of the scattergram, while the medium and large calibre fibres form a linear regression in which sheath thickness decreases with increasing calibre. The thinly myelinated fibres of small calibre may be defined as having a diameter of less than $6 \mu \mathrm{m}$ and relatively thin myelin sheaths with a g-ratio near 8.8 ; they are particularly numerous in human sural nerves where they accounted for about two thirds of the total fibre population.

The functional significance of the population of thinly myelinated fibres of small calibre is still unclear. From our present understanding of internode geometry, one would assume that these fibres myelinate relatively late, thus acquiring very short internodes with correspondingly thin sheaths. ${ }^{10}$ Late myelination between one and six months is implied by our scattergrams. The calibre below $6 \mu \mathrm{m}$ is compatible with either autonomic or afferent sensory function. Attribution to the autonomic nervous system is unlikely, in view of the abundance of these fibres in human sural nerve. Histograms of sural nerves of patients with congenital insensitivity to pain show an absence of the calibre peak corresponding to the thinly myelinated fibre population of small calibre." 12 This suggests, tentatively, that the population of thinly myelinated fibres of small calibre conducts pain. The abundance of these fibres in sural nerve may relate to the size of the cutaneous surface area supplied by this nerve.

A comparison of age groups shows that the two fibre classes cannot be separated at the age of one month. Indeed, one may surmise that the fibres present at this age are all fibres which grow to larger calibres, the small fibres entering later on. After six months, there is a separation between the two classes with a gradual increase in the thickness of their sheaths, albeit at different rates. These data confirm the well-known fact that infants and children have thinner myelin sheaths than adults. ${ }^{5}$

An advantageous feature of our method is the option either to correct automatically for noncircularity along with documentation of the extent of non-circularity for each sample, or else to print data based on the non-circular state of the fibres. The non-circularity observed in the present biopsy material was so variable among fibres and from one specimen to the next that it had to be attributed to suboptimal tissue preservation. Consideration of non-circularity is a relatively new concern in fibre morphometry. ${ }^{13}$ Many factors are known to modify the shape of fibres seen in the fixed and embedded preparation; comparison of freeze-substituted tissue with chemically fixed tissue showed that chemical fixation reduced non-circularity factors by approximately 0.2 (from 0.9 to 0.7 ). ${ }^{14}$ The physiological osmolarity of body fluids is also important. Frogs and mammals have different body fluid osmolarities; when fixed in the same medium, frogs had a noncircularity factor of 0.59 whereas rats had values of $0.85 .^{2}$ There is also evidence of changes in noncircularity of fibres induced by pathological changes 
in body fluid osmolarities. ${ }^{15}$ The osmolarities of fluids used for fixation also affect fibre shrinkage; variation of in vitro osmolarities are accompanied by commensurate variations of fibre noncircularity: 141617 lateral compression during cutting may also alter the fibre's profile. The in vivo state of the fibre, finally, is also important. Atrophy of fibres is accompanied by reduced circularity $\mathrm{y}^{18} 14$ and there are also claims that the fibres of different calibre have different non-circularities. ${ }^{1316}$ In our experience, there was no consistent trend showing selective distortions of smaller or larger fibres. ${ }^{2}$ The only distortion that is not corrected for by this program is the correction of the g-ratio to its in vivo state. ${ }^{20}$ This accounts for the changes in fibre geometry resulting from reduced interperiod width of fixed and embedded myelin. The conclusions drawn here are largely independent of this correction. Considering all these factors affecting fibre shrinkage it is appropriate to use the correction for circular profiles as a standard of reference. Correction for circular profiles enables comparison of data from different samples independent of the vagaries introduced by suboptimal tissue preservation. If there is reason to suspect in vivo changes in circularity, this can be tested in our program, but such tests would require more rigid demands on the conditions of tissue preservation than were available for our biopsy material.

Some abnormal scattergrams were added to this study to explore the method's usefulness in analysing pathological changes. Such data are tentative, as much more experience must be gathered in interpreting pathological scattergrams. One may expect that scattergrams of the g-ratio will be helpful along two lines. First, the method has a potential for demonstrating quantitatively the extent of regeneration or remyelination in a given nerve. The method is quite sensitive for the detection of minor anomalies of sheath thickness. There is evidence that regenerated fibres have permanently thinner sheaths than normal fibres, ${ }^{21}{ }^{22}$ and the same may be true for remyelinated fibres, although there are no comparable morphometric data on the latter.

Based on the experimental evidence with regenerated fibres, ${ }^{17}$ we may interpret the scattergrams of the patients in figs $8 \mathrm{a}$ and $8 \mathrm{~b}$ as showing regeneration or remyelination, an interpretation consistent with the available clinical data. The following criteria suggest regeneration: (1) a distinct upwards shift in the g-ratio (meaning thinner than normal sheaths of the regenerated fibres); (2) a slight shift to the left (meaning subtotal restitution of fibre calibres); and (3) a parabolic shape of the scattergram of fibres in the left upper quadrant (empirically found to be characteristic of immature or regenerat- ing fibre populations).

Second, the improved distinction of the two populations of fibres offers hope that preferential damage to either one or the other class of fibres may be more clearly distinguishable by this method than by the conventional calibre histograms. The difference between figs $8 \mathrm{a}$ and $8 \mathrm{~b}$ may indicate selective damage and regeneration of the small calibre fibres in fig $8 \mathrm{~b}$. The data shown for four cases of alcoholic polyneuropathy (the disease was selected arbitrarily as a reasonably defined pathological entity) suggested, at first sight, a selective loss of large fibres, beginning with those having the largest calibre. This impression may be misleading, however, as a slight loss of the thinly myelinated fibres of small calibre is not as obvious as for the more sparsely distributed large calibre fibres. Even with marked loss of fibres, there is still a near normal ratio of the two populations. Also, there was no evidence of fibres with abnormally thin sheaths (high g-ratio). Our tentative interpretation of these histograms is that alcoholic neuropathy affects fibres of all classes, and that there is no evidence of regeneration. Future studies will have to test and verify these interpretations.

The diligent assistance of Ms Sabine Ropte is acknowledged. This study was in part supported by a grant from the Deutsche Forschungsgemeinschaft (Fr 609/1-1).

\section{References}

' Zimmerman IR, Karnes JL, O'Brien PC, Dyck PJ. Imaging system for nerve and fiber tract morphometry: components, approaches, performance, and results. J Neuropathol Exp Neurol 1980;39:409-19.

${ }^{2}$ Friede RL, Beuche W. A new approach toward analyzing peripheral nerve fiber populations. I. Variance in sheath thickness corresponds to different geometric proportions of the internodes. J Neuropathol Exp Neurol 1985;44:60-72.

${ }^{3}$ Friede RL, Samorajski T. Relation between the number of myelin lamellae and axon circumference in fibers of vagus and sciatic nerves of mice. J Comp Neurol 1967; 130:223-32.

${ }^{4}$ Karnes J, Robb R, O’Brien PC, Lambert EH, Dyck PJ. Computerized image recognition for morphometry of nerve attribute to shape of sampled transverse sections of myelinated fibers which best estimates their average diameter. J Neurol Sci 1977;34:43-51.

${ }^{5}$ Schröder JM, Bohl J, Brodda K. Changes of the ratio between myelin thickness and axon diameter in the human developing sural nerve. Acta Neuropathol (Berl) 1978;43:169-78.

- Fraher JP. A quantitative study of anterior root fibres during early myelination. J Anat 1972;112:99-114.

${ }^{7}$ Hildebrand C, Hahn R. Relation between myelin sheath 
thickness and axon size in spinal cord white matter of some vertebrate species. J Neurol Sci 1978;38:42134.

* Arbuthnott ER, Boyd IA, Kalu KU. Ultrastructural dimensions of myelinated peripheral nerve fibres in the cat and their relation to conduction velocity. $J$ Physiol (Lond) 1980;308:125-57.

" Boyd IA, Kalu KU. The relation between axon size and number of lamellae in the myelin sheath for afferent fibres in groups I, II and III in the cat. J Physiol (Lond) 1973;232:31P-3P.

${ }^{10}$ Friede RL, Bischhausen R. How are sheath dimensions affected by axon caliber and internode length? Brain Res 1982;235:335-50.

" Dyck PJ, Mellinger JF, Reagan TJ, et al. Not " indifference to pain" but varieties of hereditary sensory and autonomic neuropathy. Brain 1983;106:373-90.

${ }_{12}$ Goebel HH, Veit S, Dyck PJ. Confirmation of virtual unmyelinated fiber absence in hereditary sensory neuropathy type IV. J Neuropathol Exp Neurol 1980;39:670-5.

${ }_{13}$ Arbuthnott ER, Ballard KJ, Boyd IA, Kalu KU. Quantitative study of the non-circularity of myelinated peripheral nerve fibres in the cat. $J$ Physiol (Lond) 1980;308:99-123.

14 Minwegen P, Friede RL. Conduction velocity varies with osmotically induced changes of the area of the axon's profile. Brain Res 1984;297:105-13.

15 Sugimura K, Windebank AJ, Natarajan V, Lambert EH,
Schmid HHO, Dyck PJ. Interstitial hyperosmolarity may cause axis cylinder shrinkage in streptozotocin diabetic nerve. J Neuropathol Exp Neurol 1980;39:710-21.

${ }^{16}$ Ohnishi A, O'Brien PC, Dyck PJ. Studies to improve fixation of human nerves. J Neurol Sci 1976;27:1939.

${ }^{17}$ Holland GR. The effect of buffer molarity on the size, shape and sheath thickness of peripheral myelinated nerve fibres. J Anat 1982;135:183-90.

${ }^{18}$ Gillespie MJ, Stein RB. The relationship between axon diameter, myelin thickness and conduction velocity during atrophy of mammalian peripheral nerves. Brain Res 1983;259:41-56.

${ }^{14}$ O' Neill JH, Jacobs JM, Gilliatt RW, Baba M. Changes in the compact myelin of single internodes during axonal atrophy. Acta Neuropathol (Berl) 1984;63:313-8.

${ }^{20}$ Berthold $\mathrm{C}-\mathrm{H}$, Corneliuson O, Rydmark M. Changes in shape and size of cat spinal root myelinated nerve fibers during fixation and Vestopal-W embedding for electron microscopy. J Ultrastruct Res 1982;80:23-41.

${ }^{21}$ Beuche W, Friede RL. A new approach toward analyzing peripheral nerve fiber populations. II. Foreshortening of regenerated internodes corresponds to reduced sheath thickness. J Neuropathol Exp Neurol 1985; 44:73-84.

${ }^{22}$ Ludwin SK, Maitland M. Long-term remyelination fails to reconstitute normal thickness of central myelin sheaths. J Neurol Sci 1984;64:193-8. 